\title{
General n-dimensional quadrature transform and its application to interferogram demodulation
}

\author{
Manuel Servin* \\ Centro de Investigaciones en Optica A. C., Apartado Postal 1-948, 37150 Leon, Guanajuato, Mexico \\ Juan Antonio Quiroga \\ Departamento de Optica, Universidad Complutense de Madrid, Ciudad Universitaria S/N, 28040 Madrid, Spain \\ Jose Luis Marroquin \\ Centro de Investigacion en Matematicas, Apartado Postal 402, 36000 Guanajuato, Guanajuato, Mexico
}

Received August 19, 2002; revised manuscript received January 16, 2003; accepted January 17, 2003

\begin{abstract}
Quadrature operators are useful for obtaining the modulating phase $\phi$ in interferometry and temporal signals in electrical communications. In carrier-frequency interferometry and electrical communications, one uses the Hilbert transform to obtain the quadrature of the signal. In these cases the Hilbert transform gives the desired quadrature because the modulating phase is monotonically increasing. We propose an $n$-dimensional quadrature operator that transforms $\cos (\phi)$ into $-\sin (\phi)$ regardless of the frequency spectrum of the signal. With the quadrature of the phase-modulated signal, one can easily calculate the value of $\phi$ over all the domain of interest. Our quadrature operator is composed of two $n$-dimensional vector fields: One is related to the gradient of the image normalized with respect to local frequency magnitude, and the other is related to the sign of the local frequency of the signal. The inner product of these two vector fields gives us the desired quadrature signal. This quadrature operator is derived in the image space by use of differential vector calculus and in the frequency domain by use of a $n$-dimensional generalization of the Hilbert transform. A robust numerical algorithm is given to find the modulating phase of two-dimensional single-image closed-fringe interferograms by use of the ideas put forward. (C) 2003 Optical Society of America

OCIS codes: 2650, 3180, 5050, 6160.
\end{abstract}

\section{INTRODUCTION}

Recently a number of researchers ${ }^{1-5}$ have contributed to the understanding and development of methods to estimate the modulating phase of a single-image interferogram having closed fringes. As is well understood today, this is a problem for which a unique solution does not exist; so, additional constraints must be added in order to find a unique solution. The most widely used additional constraint is to consider the modulating phase to be smooth. Using this smoothness constraint, one is able to demodulate a single fringe pattern by minimizing a nonlinear global cost function as in Ref. 3 or by locally minimizing a nonlinear cost function and then propagating its solution by following the fringes of the interferogram as in the case of the regularized phase-tracking (RPT) algorithm. $^{2}$ In the RPT case the local phase and frequency are estimated simultaneously.

The problem of finding the quadrature signal of a single closed-fringe interferogram may also be achieved by factorizing the whole task into two separate problems, namely, finding the orientation of the fringes and their local frequency. As far as we know, this was first proposed by Marroquin et al., ${ }^{4}$ who proposed two separate cost functions to obtain the orientation and the local frequency independently. The phase-estimation problem may also be factorized by use of the RPT by scanning the interferogram, following the path defined by its fringes. ${ }^{2}$ By doing this, one is able to somewhat decouple the local phase estimation (which is almost constant along the fringes of the image) from its local frequency (fringe orientation). This two-dimensional (2D) scanning strategy is shown to be more robust than a scanning strategy independent of the form of the interferogram's fringes, such as a row-byrow scanning.

On the other hand, efforts have been made for estimating the modulating phase of a single-image closed-fringe interferogram by the use of a linear operator. ${ }^{1,5}$ This seems to be a very good approach, since if it were possible to find such an operator, this process would be as easy as demodulating a carrier-frequency interferogram. ${ }^{6}$ Unfortunately, as we show in this paper, such a linear system does not exist. In contrast, what does exist ${ }^{5}$ is a linear operator that gives the quadrature signal in a direct way, once and only once; the orientation of the fringes is already estimated (which is not a trivial process).

The first well-known study that uses a linear operator (the Hilbert transform) to obtain the modulating phase of a single-image closed-fringe interferogram was made by Kreis. ${ }^{1}$ He used a 2D generalized Hilbert operator $H_{2}{ }^{\prime}$ $\{\cdot\}$ to find the modulating phase of the interferogram. 
As we will see in this paper, the trouble with this approach is twofold. First, the recovered phase has spurious ringing effects along paths where the magnitude of the phase gradient is close to zero, owing to the hard discontinuity of the $H_{2}^{\prime}\{\cdot\}$ operator at the origin. Second, the recovered phase is always monotonically increasing even though the actual modulating phase may be nonmonotonic.

In this paper we present a quadrature operator that is composed of two vector fields; one is related to the image gradient normalized with respect to the local frequency magnitude, and the other, which we call $\mathbf{n}_{\phi}$, is related to the orientation angle of the fringes of the interferogram. We also present examples of the application of this operator to the demodulation of computer-simulated fringe patterns and to an experimentally obtained interferogram. Finally, we compare the performance of our numerical algorithm with the vortex operator proposed by Larkin et $a l .{ }^{5}$

\section{ONE-DIMENSIONAL QUADRATURE TRANSFORM}

The Hilbert transform in one dimension, $H_{1}\{\cdot\}$, is a very useful mathematical tool to obtain the quadrature of a single-frequency sinusoidal signal. According to Ref. 7, the Hilbert transforms of cosine and sine functions with a linear increasing phase are

$$
\begin{aligned}
& H_{1}\left[\cos \left(\omega_{0} x\right)\right]=-\sin \left(\omega_{0} x\right) \text { for } \omega_{0}>0 ; \\
& H_{1}\left[\sin \left(\omega_{0} x\right)\right]=\cos \left(\omega_{0} x\right)
\end{aligned}
$$

because of this, we may have the (wrong) impression that the Hilbert transform $H_{1}\{\cdot\}$ always gives the quadrature of a cosine signal. The transforming properties of the Hilbert operator become clear by looking at the form of the frequency response of $H_{1}\{\cdot\}$ as applied to a real function $g(x)=\cos [\phi(x)]$, which is ${ }^{7}$

$$
\mathcal{F}\left\{H_{1}[g(x)]\right\}=-i \operatorname{sign}(u) \hat{g}(u)=-i \frac{u}{|u|} \hat{g}(u),
$$

where the Fourier transform is represented by $\mathcal{F}\{\cdot\}$ and

$$
\hat{g}(u)=\mathcal{F}\{g(x)\} \text {. }
$$

The Hilbert transform still renders the expected result (the quadrature signal) when it is applied to a more complicated carrier-frequency signal, that is,

$$
H_{1}\left\{\cos \left[\omega_{0} x+\psi(x)\right]\right\}=-\sin \left[\omega_{0} x+\psi(x)\right],
$$

provided that the local frequency does not change sign, i.e.,

$$
\omega_{0}+\frac{\mathrm{d} \psi(x)}{\mathrm{d} x}>0, \quad \forall x .
$$

In this case this cosine signal has its two spectral lobes well separated, and the $H_{1}\{\cdot\}$ operator may be used to recover the modulating phase $\psi(x)$ of the signal. This is the reason why the Fourier method is so widely used to find the modulating phase of carrier-frequency interferograms.
Unfortunately, Eq. (4) will not hold if condition (5) is not satisfied, i.e., if the phase-modulating signal is not a monotonically increasing function of $x$. As is demonstrated in Section 3, without a carrier, one needs to know the sign of the local frequency to obtain the expected quadrature signal. Therefore, in general, to obtain the one-dimensional (1D) quadrature of $\cos (\phi)$, one needs to use the following formula, which relates the 1D quadrature transform $Q_{1}\{\cdot\}$ to the 1D Hilbert transform $H_{1}\{\cdot\}$,

$$
Q_{1}\{\cos (\phi)\}=\frac{\mathrm{d} \phi / \mathrm{d} x}{|\mathrm{~d} \phi / \mathrm{d} x|} H_{1}\{\cos (\phi)\}=-\sin (\phi),
$$

where the $x$ dependence of $\phi(x)$ was omitted for clarity. The result of applying $Q_{1}\{\cdot\}$ is a reduction to $H_{1}\{\cdot\}$ only when the local frequency $\omega=\mathrm{d} \phi / \mathrm{d} x$ of the signal is everywhere greater than zero, so that the ratio $\omega /|\omega|$ equals 1 over the whole domain of interest. In general, to obtain the quadrature signal of the cosine of a nonmonotonic function $\phi(x)$, one needs to know the sign of the local spatial frequency to correct the sign of the signal obtained by the application of the Hilbert transform.

\section{TWO-DIMENSIONAL QUADRATURE TRANSFORM FOR CARRIER-FREQUENCY INTERFEROGRAMS}

A straightforward generalization for the 1D Hilbert transform has been used in optical signal processing as a component of the Schlieren phase analysis method. ${ }^{8}$ This $2 \mathrm{D}$ Hilbert transform generalization is also the one used by Kreis $^{1}$ to find the modulating phase of a closed-fringe interferogram.

To describe it, let us start by considering a 2D fringe pattern with carrier frequency. This is normally represented as

$$
I_{a}(x, y)=a(x, y)+b(x, y) \cos \left[\psi(x, y)+u_{0} x+v_{0} y\right] .
$$

This equation represents a fringe pattern $I_{a}(x, y)$ that depends on a $2 \mathrm{D}$ phase $\psi(x, y)$ plus a phase plane. The function $a(x, y)$ is a low-frequency signal that represents the background illumination. The function $b(x, y)$ is also a low-frequency signal that represents the slowly varying contrast of the fringes. The two carrier frequencies $u_{0}$ and $v_{0}$ are assumed to be greater than the maximum spatial frequency of the modulating signal along the $x$ and $y$ directions, that is,

$$
u_{0}>\left|\frac{\partial \psi(x, y)}{\partial x}\right|, \quad v_{0}>\left|\frac{\partial \psi(x, y)}{\partial y}\right|, \quad \forall(x, y) .
$$

Now let us assume that we can eliminate the background illumination $a(x, y)$, so our fringe pattern will be reduced to

$$
I(x, y)=b(x, y) \cos \left[\psi(x, y)+u_{0} x+v_{0} y\right],
$$

and this fringe pattern may be rewritten as 


$$
\begin{aligned}
I(x, y)= & \frac{b(x, y)}{2}\left(\exp \left\{i\left[-\psi(x, y)-u_{0} x-v_{0} y\right]\right\}\right. \\
& \left.+\exp \left\{i\left[\psi(x, y)+u_{0} x+v_{0} y\right]\right\}\right) .
\end{aligned}
$$

The Fourier transform of this signal may be represented as

$$
\hat{I}(u, v)=\frac{1}{2}\left[f_{-}(u, v)+f_{+}(u, v)\right],
$$

where $f_{+}(u, v)$ and $f_{-}(u, v)$ are the Fourier transforms of the corresponding complex exponential terms multiplied by the contrast function $b(x, y)$. Because of conditions (8), this signal is composed of two well-separated spectral lobes, corresponding to the signals $f_{+}(u, v)$, which lies exclusively in the positive side of the half-plane $u_{0} u+v_{0} v$ $=0$, and $f_{-}(u, v)$, which lies in the negative side. One may obtain the quadrature of the interferogram by filtering this signal using the following operator:

$$
\begin{aligned}
H_{2}^{\prime}\{I(x, y)\} & =-b(x, y) \sin \left[\psi(x, y)+u_{0} x+v_{0} y\right] \\
& =\mathcal{F}^{-1}\left\{\frac{-i \omega_{0} \cdot \mathbf{q}}{\left|\omega_{0} \cdot \mathbf{q}\right|}\left[f_{+}(u, v)+f_{-}(u, v)\right]\right\},
\end{aligned}
$$

where

$$
\omega_{0}=u_{0} \mathbf{e}_{1}+v_{0} \mathbf{e}_{2}, \quad \mathbf{q}=u \mathbf{e}_{1}+v \mathbf{e}_{2},
$$

where $\mathbf{e}_{1}$ and $\mathbf{e}_{2}$ are the unit vectors along the $u$ and $v$ spectral coordinates. The result of applying this $2 \mathrm{D}$ form of the Hilbert transform $H_{2}{ }^{\prime}\{\cdot\}$ to the fringe pattern coincides with the expected quadrature signal, because the input interferogram has its two spectral lobes well separated and the $H_{2}^{\prime}\{\cdot\}$ transform is reduced in one dimension, to the one given in Section 2. If this spectral separation condition is not satisfied, however, the result of applying $H_{2}^{\prime}\{\cdot\}$ will not give the expected quadrature signal.

The Schlieren transform $S\{\cdot\}$ uses $H_{2}{ }^{\prime}\{\cdot\}$ in the following way:

$$
\begin{aligned}
S\{\hat{I}(u, v)\} & =\frac{1}{2}\left[1+H_{2}{ }^{\prime}\{\hat{I}(u, v)\}\right] \\
& =U(u, v)+i V(u, v),
\end{aligned}
$$

where the $(u, v)$ space is the Fourier transform space of the $\mathbf{R}^{2}=(x, y)$ plane. In the optical laboratory this filter $S\{\cdot\}$ is easily implemented simply by blocking out (with a knife edge, for example) half of the spectral domain along the line given by $\omega_{0} \cdot \mathbf{q}=0$. The estimated phase $\psi(x, y)$ is then obtained from

$$
\psi(x, y)+u_{0} x+v_{0} y=\tan ^{-1}\left[\frac{\operatorname{Im}(A)}{\operatorname{Re}(A)}\right],
$$

where

$$
A(x, y)=\mathcal{F}^{-1}\{U(u, v)+i V(u, v)\} .
$$

Note that, because of conditions (8), the phase obtained by this relation is always monotonic owing to the phase carrier. Therefore, when the Schlieren operator $S\{\cdot\}$ is used to obtain the phase of a closed-fringe interferogram, the estimated phase does not represent the actual nonmonotonic modulating phase; also, the estimated phase in this case has serious spurious ringing effects along the path where this phase is stationary. However, in the case of carrier-frequency-interferogram phase demodulation by use of the Schlieren operator, $S\{\cdot\}$ works perfectly well, and its estimated phase is free of undesirable phase distortions.

\section{GENERAL $n$-DIMENSIONAL QUADRATURE TRANSFORM}

In this section we will present a general $n$-dimensional quadrature transform that works well for closed fringes, as well as for carrier-frequency interferograms. Since it works for any dimension, it may also be useful for processing spatiotemporal (three-dimensional) fringe patterns that arise in fast transient optical metrology, for example. Moreover, the result of this section will also permit us to obtain as special case the $1 \mathrm{D}$ result stated in Section 2.

The aim of an $n$-dimensional quadrature operator $Q_{n}$ $\{\cdot\}$ is to transform a given fringe pattern into its quadrature, which may be represented by

$$
Q_{n}\{b(\mathbf{r}) \cos [\phi(\mathbf{r})]\}=-b(\mathbf{r}) \sin [\phi(\mathbf{r})],
$$

where $\mathbf{r}=\left(x_{1}, \ldots, x_{n}\right)$ is the $n$-dimensional vector position. Using this quadrature signal, one can easily determine the phase $\phi(\mathbf{r})$ modulo $2 \pi$ over the whole domain of interest.

The first step toward obtaining the quadrature signal is to obtain the gradient of the fringe pattern, which is

$$
\nabla I(\mathbf{r})=\cos [\phi(\mathbf{r})] \nabla b(\mathbf{r})+b(\mathbf{r}) \nabla\{\cos [\phi(\mathbf{r})]\} .
$$

Given that in most practical situations the contrast $b(\mathbf{r})$ is a low-frequency signal, the first term in this equation may be negligible with respect to the second one, so that one obtains

$$
\nabla I(\mathbf{r}) \approx b(\mathbf{r}) \nabla\{\cos [\phi(\mathbf{r})]\} ;
$$

hereafter we will assume this approximation is valid, so the approximation sign will be replaced by an equal sign. Of course, for the special case of a constant contrast $b(\mathbf{r})=b_{0}$, the above mathematical relation is exact. Applying the chain rule for differentiation, we obtain

$$
\nabla I(\mathbf{r})=-b(\mathbf{r}) \sin [\phi(\mathbf{r})] \nabla \phi(\mathbf{r}) .
$$

If it were possible to know the actual sign and magnitude of the local frequency $\nabla \phi(\mathbf{r})$, one could use this information as

$$
\nabla I(\mathbf{r}) \cdot \nabla \phi(\mathbf{r})=-b(\mathbf{r}) \sin [\phi(\mathbf{r})]|\nabla \phi(\mathbf{r})|^{2},
$$

and the quadrature signal would be obtained by dividing both sides of this equation by the squared magnitude of the local frequency $|\nabla \phi(\mathbf{r})|^{2}$ :

$$
\begin{aligned}
Q_{n}\{b(\mathbf{r}) \cos [\phi(\mathbf{r})]\} & =\frac{\nabla \phi(\mathbf{r})}{|\nabla \phi(\mathbf{r})|^{2}} \cdot \nabla I(\mathbf{r}) \\
& =-b(\mathbf{r}) \sin [\phi(\mathbf{r})] .
\end{aligned}
$$

We start by noticing that the equation for $Q_{n}\{\cdot\}$ is a little bit tricky because, as far as we know, there is no linear system that can be applied to our fringe pattern $I(\mathbf{r})$ to give us $\nabla \phi(\mathbf{r})$ in a direct way. In the following we pro- 
pose some techniques to calculate the gradient of the modulating phase. Equation (22) may be rewritten as

$$
\begin{aligned}
Q_{n}\{b(\mathbf{r}) \cos [\phi(\mathbf{r})]\} & =\frac{\nabla \phi(\mathbf{r})}{|\nabla \phi(\mathbf{r})|} \cdot \frac{\nabla I(\mathbf{r})}{|\nabla \phi(\mathbf{r})|} \\
& =\mathbf{n}_{\phi} \cdot \frac{\nabla I(\mathbf{r})}{|\nabla \phi(\mathbf{r})|},
\end{aligned}
$$

where $\mathbf{n}_{\phi}=\mathbf{n}_{\phi}(\mathbf{r})$ is a unit vector normal to the corresponding isophase contour, which points in the direction of $\nabla \phi$. This mathematical relation is the desired quadrature operator and the main result of this paper. In the remainder of the paper we will discuss some properties of this operator as well as several techniques that may be used to numerically calculate it as a computer program that can be used to demodulate single- or multiple-closed fringe interferograms.

In two dimensions, the vector $\mathbf{n}_{\phi}$ points in the direction $\theta_{2 \pi}$, which we call the fringe orientation angle, which is given by

$$
\tan \left[\theta_{2 \pi}(x, y)\right]=\frac{\omega_{y}(x, y)}{\omega_{x}(x, y)},
$$

with

$$
\omega_{x}=\frac{\partial \phi(x, y)}{\partial x}, \quad \omega_{y}=\frac{\partial \phi(x, y)}{\partial y} .
$$

From this relation, one can obtain the cosine and sine of the local fringe orientation:

$$
\begin{aligned}
\cos \left[\theta_{2 \pi}(x, y)\right] \mathbf{i}+\sin [ & \left.\theta_{2 \pi}(x, y)\right] \mathbf{j} \\
& =\frac{\omega_{x} \mathbf{i}}{\left(\omega_{x}{ }^{2}+\omega_{y}{ }^{2}\right)^{1 / 2}}+\frac{\omega_{y} \mathbf{j}}{\left(\omega_{x}{ }^{2}+\omega_{y}{ }^{2}\right)^{1 / 2}} \\
& =\frac{\nabla \phi(x, y)}{|\nabla \phi(x, y)|} .
\end{aligned}
$$

This relation may be generalized to higher dimensions. For example, in three dimensions, one obtains

$$
\begin{aligned}
\mathbf{n}_{\phi}= & \frac{\nabla \phi(x, y, z)}{|\nabla \phi(x, y, z)|} \\
= & \cos \alpha_{2 \pi}(x, y, z) \mathbf{i}+\cos \beta_{2 \pi}(x, y, z) \mathbf{j} \\
& \quad+\cos \gamma_{2 \pi}(x, y, z) \mathbf{k},
\end{aligned}
$$

where $\mathbf{i}, \mathbf{j}$, and $\mathbf{k}$, are the unit vectors along the $x, y$, and $z$ coordinates and $\alpha, \beta$, and $\gamma$ are the direction cosines of the normal vector $\mathbf{n}_{\phi}$. As can be seen, the use of direction cosines permits one to extend the concept of $2 \mathrm{D}$ fringe orientation to higher dimensions. If we know the following property of direction cosines,

$$
\begin{aligned}
\cos ^{2}\left[\alpha_{2 \pi}(x, y, z)\right]+\cos ^{2}[ & \left.\beta_{2 \pi}(x, y, z)\right] \\
+ & \cos ^{2}\left[\gamma_{2 \pi}(x, y, z)\right]=1,
\end{aligned}
$$

any two direction cosines or, equivalently, two orientation angles, are needed to obtain the third one. In general, in an $n$-dimensional space, one would need to obtain the orientation of $(n-1)$ angles. Conversely, knowing the magnitude and sign of the phase gradient, one can find the direction cosines of the fringe's orientation. Unfortunately, there is no direct way (e.g., a linear transformation) to obtain $\mathbf{n}_{\phi}$ from a single-image fringe pattern containing closed fringes. That is because there is a fundamental ambiguity in the global sign of the recovered phase. So, in general, one decides arbitrarily the sign of the phase at a given seed point, and afterward one propagates that phase solution throughout the entire interferogram.

One can see that the quadrature operator of Eq. (23) reduces, in one dimension, to the form for $Q_{1}\{\cdot\}$ stated in Section 2. In Subsection 4.A we give a numerical technique for calculating the quadrature operator of a closedfringe interferogram $I(x, y)$. In Subsection 4.B we show another alternative for calculating $\nabla I /|\nabla \phi|$ as a linear operator whose Fourier transform corresponds to $n$ Reisz transforms ${ }^{9}$ along each spectral coordinate. We denote this operator by $\mathbf{H}_{n}\{\cdot\}$, given that this is another possible generalization of the 1D Hilbert transform. This generalization of $H_{1}\{\cdot\}$ is different from the 2D generalization used in the Schlieren method mentioned in Section 3.

\section{A. Numerical Method to Calculate the Quadrature of a} Two-Dimensional Signal $\boldsymbol{Q}_{\mathbf{2}}\{\boldsymbol{I}(\boldsymbol{x}, \boldsymbol{y})\}$

In this subsection we will use the ideas put forward in this paper to propose a practical numerical method to demodulate a single closed-fringe interferogram. For the reader's convenience, let us first recall the form of our quadrature operator in two dimensions:

$$
Q_{2}\{I(x, y)\}=\frac{\nabla \phi(x, y)}{|\nabla \phi(x, y)|} \cdot \frac{\nabla I(x, y)}{|\nabla \phi(x, y)|}
$$

we can see that there are several ways to calculate $Q_{2}$ $\{\cdot\}$. The orientation term $\nabla \phi /|\nabla \phi|$, as we mentioned, is by far the most difficult term to estimate. To calculate $\nabla \phi /|\nabla \phi|$, we recommend using the Quiroga et al. ${ }^{10}$ technique outlined in Subsection 4.C. In contrast, the term $\nabla I /|\nabla \phi|$ is easier to compute. A possible way to find $\nabla I /|\nabla \phi|$ is to estimate $|\nabla \phi|$ and $\nabla I$ directly from the fringe pattern. Another possibility, which is very easy to implement and is the one used in this subsection, is to calculate just $\nabla I$ instead of calculating $\nabla I /|\nabla \phi|$ by use of centered first-order differences. Obviously, $\nabla I$ and $\nabla I /|\nabla \phi|$ will differ when $|\nabla \phi| \neq 1$.0, so a final adjustment will be necessary. By doing this, one obtains

$$
\mathbf{n}_{\phi}(x, y) \cdot \nabla I(x, y)=|\nabla \phi(x, y)| \sin [\phi(x, y)],
$$

where we have assumed that the fringe pattern is normalized so that $b(x, y)=1$ and its fringe orientation modulo $2 \pi(\nabla \phi /|\nabla \phi|)$ has been already estimated. We can see that the signal in the left-hand side of Eq. (30) is proportional to the quadrature signal; therefore the phase obtained by

$$
\begin{aligned}
\phi^{0}(x, y) & =\tan ^{-1}\left[\frac{\mathbf{n}_{\phi}(x, y) \cdot \nabla I(x, y)}{I(x, y)}\right] \\
& =\tan ^{-1}\left\{\frac{|\nabla \phi(x, y)| \sin [\phi(x, y)]}{\cos [\phi(x, y)]}\right\}
\end{aligned}
$$


may have a small phase error over the $2 \mathrm{D}$ regions where $|\nabla \phi| \neq 1$, but, fortunately, it is in the correct branch of the $\tan ^{-1}(\cdot)$ function. The correct phase value may therefore be found as the solution to the nonlinear equation

$$
f(x, y)=\cos [\phi(x, y)]-I(x, y)=0,
$$

which is closest to the phase value $\phi^{0}(x, y)$ obtained above. The value for $\phi^{0}(x, y)$ is used as an initial guess in an iterative algorithm that moves closer to the real modulating phase. This may be done pixel by pixel by use of the very efficient iterative Halley method, ${ }^{11}$ which is an improved version of the Newton-Raphson technique; the iteration is given by

$$
\begin{array}{r}
\phi^{k+1}(x, y)=\phi^{k}(x, y) \\
-\frac{2 f(x, y) f_{\phi}(x, y)}{2 f_{\phi}^{2}(x, y)+f_{\phi \phi}(x, y) f(x, y)}, \\
\quad k=0,1,2, \ldots,
\end{array}
$$

where $f_{\phi}(x, y)$ and $f_{\phi \phi}(x, y)$ are the first and second derivatives of $f(x, y)$ with respect to $\phi$, which gives

$$
\begin{aligned}
& \phi^{k+1}(x, y) \\
&= \phi^{k}(x, y) \\
&+ \frac{2 f(x, y) \sin \left[\phi^{k}(x, y)\right]}{2 \sin ^{2}\left[\phi^{k}(x, y)\right]-\cos \left[\phi^{k}(x, y)\right] f(x, y)}, \\
& k=0,1,2, \ldots .
\end{aligned}
$$

This recursive formula is very stable and extremely efficient given that the value $\phi^{k+1}(x, y)$ approaches the searched for solution $\phi(x, y)$; the formula triples the number of accurate significant figures at each iteration. So, on average, approximately two iterations per pixel are needed to correct the estimated phase.

One might think that to solve Eq. (32) one needs only to evaluate

$$
\phi(x, y)=\cos ^{-1}[I(x, y)] .
$$

The problem with this solution is that our resultant $\phi(x, y)$ will be obtained modulo $\pi$, and this is not what we are looking for, so we end up at the beginning of our problem. In contrast, what we need is the nonmonotonic solution for $\phi(x, y)$ modulo $2 \pi$, which is obtained by the proposed method.

\section{B. Fourier Transform Method to Calculate the Vector Field $\nabla I /|\nabla \phi|$}

In this subsection we show how the vector field $\nabla I /|\nabla \phi|$ may also be calculated using the Fourier transform. This Fourier operator acting on $I(x, y)$ may be seen as a $n$-dimensional generalization of the 1D Hilbert transform. We will show this in two dimensions, but it may be readily extended to $n$ dimensions. We start by writing a mathematical generalization of the 2D Hilbert transform, and we then demonstrate that this Fourier-transformed kernel is approximately equal to our operator $\nabla I /|\nabla \phi|$. The 2D generalization of the Hilbert transform that we will use is

$$
\begin{aligned}
\mathbf{H}_{2}\{b \cos (\phi)\}= & \mathcal{F}^{-1}\left\{\left[\frac{-i u}{\left(u^{2}+v^{2}\right)^{1 / 2}} \mathbf{e}_{1}\right.\right. \\
& \left.\left.+\frac{-i v}{\left(u^{2}+v^{2}\right)^{1 / 2}} \mathbf{e}_{2}\right] \mathcal{F}\{b \cos (\phi)\}\right\},
\end{aligned}
$$

where we define $\mathcal{F}^{-1}\left(a \mathbf{e}_{1}+b \mathbf{e}_{2}\right)=\mathcal{F}^{-1}(a) \mathbf{e}_{1}$ $+\mathcal{F}^{-1}(b) \mathbf{e}_{2}$. Note that the operator $\mathbf{H}_{2}\{\cdot\}$ is a realvalued vector field, since the functions $-i u \mathcal{F}[b \cos (\phi)]$ and $-i v \mathcal{F}[b \cos (\phi)]$ are Hermitian. This linear operator is the vectorial sum of two Reisz transforms ${ }^{9}$ along each coordinate in the spectral space. This $\mathbf{H}_{2}\{\cdot\}$ operator is equivalent to the spiral operator presented by Larkin et al. ${ }^{5}$ when vectors $\mathbf{e}_{1}$ and $\mathbf{e}_{2}$ are replaced by 1 and $i$, respectively (note that if this substitution is performed $\mathbf{H}_{2}\{\cdot\}$ would be complex valued, but it is not a vector field). We have found, however, that from a computational viewpoint, Eq. (36) is better behaved because of the numerical cross talk between real and imaginary parts that takes place when a single Fourier transform is performed. Equation (36) may be rewritten as

$$
\mathbf{H}_{2}\{I(x, y)\}=\mathcal{F}^{-1}\left\{\frac{\mathcal{F}[\nabla I(x, y)]}{\left(u^{2}+v^{2}\right)^{1 / 2}}\right\} .
$$

Using the approximation stated in Appendix A, we may write Eq. (37) as

$$
\mathbf{H}_{2}\{I(x, y)\} \approx \mathcal{F}^{-1}\left(\mathcal{F}\left\{\frac{\nabla I(x, y)}{\left[\omega_{x}^{2}(x, y)+\omega_{y}{ }^{2}(x, y)\right]^{1 / 2}}\right\}\right),
$$

which finally gives

$$
\mathbf{H}_{2}\{I(x, y)\} \approx \frac{\nabla I(x, y)}{|\nabla \phi(x, y)|} ;
$$

given that this relation is stated in vectorial form, it is valid in any dimension in Euclidean space. As can be seen from Appendix A, the approximation used to transform Eq. (37) into relation (38) is more accurate when the modulating phase $\phi(x, y)$ varies slowly. The result stated in relation (39) may be used to demonstrate that the vortex operator ${ }^{5}$ is an approximation to the quadrature transform $Q_{2}\{\cdot\}$ proposed in this paper. As shown in Appendix A, this approximation is valid only for a smooth modulating phase.

According to what has been presented in this subsection, we have, at least, the following two alternative ways to calculate this particular $n$-dimensional generalization of the Hilbert transform; these are

$$
\begin{aligned}
& \mathbf{H}_{n}\{I(\mathbf{r})\} \approx \frac{\nabla I(\mathbf{r})}{|\nabla \phi(\mathbf{r})|}, \\
& \mathbf{H}_{n}\{I(\mathbf{r})\} \approx \mathcal{F}^{-1}\left[\frac{-\mathbf{q}}{|\mathbf{q}|} \hat{I}(\mathbf{u})\right],
\end{aligned}
$$

where $\mathbf{u}$ is a point in the $n$-dimensional spectral space. The variables $\mathbf{r}$ and $\mathbf{q}$ are the position vectors in the spectral and the image domains, respectively, which are given by 


$$
\mathbf{r}=\left(x_{1}, \ldots, x_{n}\right), \quad \mathbf{q}=\left(u_{1}, \ldots, u_{n}\right) .
$$

Note that this $\mathbf{H}_{n}\{\cdot\}$ generalization transform also reduces to the 1D Hilbert transform when it is applied to a 1D signal.

\section{Numerical Method to Calculate the Vector Field $n_{\phi}(x, y)$}

As we have shown in Subsection 4.B, the most critical part in computing the quadrature of a fringe pattern $I(\mathbf{r})$ is the estimation of the fringe orientation field $\mathbf{n}_{\phi}$ $=\nabla \phi|| \nabla \phi \mid$. As we mentioned, it is impossible to find a linear system to calculate this vector field directly from the interferogram. The reason is that $\phi(x, y)$ is wrapped by the observation process $[\cos (\phi)]$, so we do not have direct access to it (otherwise, our problem would be solved). What we do have access to, from a single-image interferogram $I(x, y)$, is the fringe orientation modulo $\pi$. This orientation angle must be unwrapped to obtain the searched-for orientation modulo $2 \pi$.

For the reader's convenience, in this subsection we briefly outline the main ideas behind the technique proposed by Quiroga et $a l .{ }^{10}$ to unwrap the fringe orientation angle modulo $\pi$ to obtain the required orientation angle modulo $2 \pi$. The orientation angle modulo $\pi$ may be easily found from the interferogram irradiance by

$$
\tan \left[\theta_{\pi}(x, y)\right]=\frac{\partial I(x, y) / \partial y}{\partial I(x, y) / \partial x} .
$$

This formula is valid, provided that the fringe pattern $I(x, y)$ has been previously normalized, which means that the amplitude-modulation term $b(x, y)$ is almost constant over the whole region of interest.

The relation between the fringe orientation angle $\theta_{\pi}$ modulo $\pi$ with the modulo $2 \pi$ orientation angle $\theta_{2 \pi}$ is

$$
\theta_{\pi}=\theta_{2 \pi}+k \pi
$$

where $k$ is an integer. Using this relation, one may write

$$
2 \theta_{\pi}=2 \theta_{2 \pi}+2 k \pi=W\left(2 \theta_{2 \pi}\right) .
$$

This result states that the value for $2 \theta_{\pi}$ is indistinguishable from that for the wrapped angle $W\left(2 \theta_{2 \pi}\right)$. Therefore it is possible to obtain the unwrapped $\theta_{2 \pi}$ by unwrapping $W\left(2 \theta_{\pi}\right)$ and finally dividing the unwrapped signal $2 \theta_{2 \pi}$ by 2 .

However, the unwrapping of $2 \theta_{\pi}$ cannot be carried out by standard path-independent techniques ${ }^{12}$ because in the presence of closed fringes it is not a consistent field: It may be easily verified that the sum of wrapped differences of $2 \theta_{\pi}$ along any closed path that contains a center of closed fringes will be nonzero (in fact, it will be close to $4 \pi$ ), which is a sufficient condition for path dependency in the unwrapping process. ${ }^{13}$ One useful path-dependent strategy is to unwrap the signal $2 \theta_{\pi}$ following the fringes of the interferogram. In this way, one normally encircles (avoids) the discontinuity point at the center of the closed fringes until its surroundings has been unwrapped. ${ }^{10}$ Because of the large noise normally encountered in practice for $2 \theta_{\pi}$ (owing to the ratio of two derivatives), one must use robust path-dependent strategies. The algorithm that best fits these requirements is the unwrapping algorithm based on the RPT. ${ }^{14}$ A detailed account of the fringe's orientation angle unwrapping, along with interesting examples, is given by Quiroga et al. in Ref. 10 .

\section{DISCUSSION}

The main contribution of this paper is the expression of the quadrature operator as the dot product of two vector fields [Eq. (23)]. A particular case in which this idea is implicit is the Vortex transform proposed by Larkin, ${ }^{5}$ although in that case it is treated as the product of two complex-valued fields. Here we generalize it in an important way: By giving explicit vectorial formulas for the characterization of these two fields, we not only make the extension to higher dimensions direct but also open the possibility for using other methods for the computation of each one of these components, which may be more efficient or exhibit a better behavior or both. Thus we show in this paper that there are several ways to compute the vector field $\nabla I /|\nabla \phi|$. One possibility is estimating $\nabla I$ and $|\nabla \phi|$ separately and then combining their outcome. A second one is to use $\nabla I$ as a coarse approximation to this field and then correct the phase estimate, using a pixelwise iterative procedure (as in Subsection 4.A). A third way is the use of Fourier transform techniques (as in Subsection 4.B), which in the special $2 \mathrm{D}$ case is equivalent to Larkin et al.'s spiral filter. ${ }^{5}$ We believe, however, that the vectorial representation is preferable, both from a theoretical viewpoint (since the generalization to higher dimensions becomes direct) and from a computational one; Eq. (36) is better behaved because of the numerical cross talk between real and imaginary parts that takes place when a single Fourier transform is performed. As we will show in Section 5, the best results are obtained by the space-domain method of Subsection 4.A. Because of its mathematical form, the $\mathbf{H}_{2}\{\cdot\}$ operator given by relation (39) gives unreliable results in places where the local frequency magnitude is close to zero; in contrast, these distortions are automatically and efficiently corrected by the pixelwise Halley iterations of Eq. (34).

The most difficult part of the whole process is the estimation of the orientation angle modulo $2 \pi$ from its orientation modulo $\pi$, and this last step is the main reason why the estimation of the quadrature of single-image interferograms with closed fringes is most difficult. In any case, it seems hopeless to reach the phase-demodulation robustness of phase stepping or carrier-frequency interferometry when a single closed-fringe interferogram is analyzed; the reason is that we have infinitely many solutions compatible with the observations in this case.

One might be tempted to say that the quadrature operator $Q_{n}\{\cdot\}$ proposed here, as well as the vortex operator in Ref. 5 are direct methods for estimating the quadrature of a single image with closed fringes. We feel that the word "direct" is not accurate here because it might be interpreted as meaning that, with these techniques, one may demodulate a single closed-fringe interferogram as easily as in the case of carrier-frequency interferometry, but unfortunately this is not the case. The reasons are that the vortex operator, as well as the quadrature operator presented in this paper, readily gives the quadrature of the signal once and only once, the orientation of the 
fringes modulo $2 \pi$, and hence $\mathbf{n}_{\phi}$, has been determined, and the estimation of the fringe orientation modulo $2 \pi$ from a single closed-fringe pattern is a process that is far from direct.

Once $\mathbf{n}_{\phi}$ is known, one may even use the form of the $2 \mathrm{D}$ Hilbert transform $H_{2}{ }^{\prime}\{\cdot\}$ contained in the Schlieren operator $S\{\cdot\}$ to obtain another 2D quadrature operator $Q_{2}^{\prime}\{\cdot\}$ :

$$
Q_{2}^{\prime}\{\cos (\phi)\}=\frac{\omega_{0} \cdot \mathbf{n}_{\phi}}{\left|\omega_{0} \cdot \mathbf{n}_{\phi}\right|} H_{2}^{\prime}\{\cos (\phi)\}=-\sin (\phi),
$$

where the vector $\omega_{0}=u_{0} \mathbf{i}+v_{0} \mathbf{j}$ is the chosen filtering direction that is used by the operator $H_{2}{ }^{\prime}\{\cdot\}$. This formula for $Q_{2}^{\prime}\{\cdot\}$ is just a 2D application of the 1D quadrature operator presented in Section 2 . It has, however, a drawback: The $H_{2}^{\prime}\{\cdot\}$ operator severely distorts the quadrature signal obtained because of the hard spectral discontinuity of the associated filter. As one may see, the $n$-dimensional quadrature operator is not unique; in our opinion, $Q_{2}^{\prime}\{\cdot\}$ is more difficult to evaluate numerically than $Q_{2}\{\cdot\}$. The fact that several quadrature operators $Q$ are possible, however, opens the possibility of finding other quadrature operators better suited for numerical estimation.

Finally, one may realize that the solution to the problem of demodulating a single interferogram in two dimensions may also be obtained directly from Eq. (20) [with $b(\mathbf{r})=1.0$ ], i.e., by solving the following set of nonlinear coupled partial differential equations,

$$
\begin{aligned}
& \sin (\phi) \frac{\partial \phi}{\partial x}+I_{x}=0, \\
& \sin (\phi) \frac{\partial \phi}{\partial y}+I_{y}=0,
\end{aligned}
$$

where $I_{x}(x, y)$ and $I_{y}(x, y)$ are the partials of the interferogram $I(x, y)$ with respect to coordinates $x$ and $y$, the amplitude modulation of the interferogram $b(x, y)$ $\approx 1.0$ has been normalized, and the spatial $(x, y)$ dependence was omitted for clarity purposes. The form of this set of partial differential equations may stimulate our imagination to find alternative ways or systems to demodulate interferograms. Among these demodulating systems, one might think of powerful nonlinear finiteelement methods to find an approximate solution to this set of equations.

\section{EXPERIMENTAL RESULTS}

We begin by considering the noiseless computergenerated fringe pattern of Fig. 1(a). We show this noisefree example to appreciate the form of the signals involved in the estimation of the modulating phase $\phi(x, y)$ of a single-image interferogram containing closed fringes. Figure 1(a) shows the input fringe pattern, and Fig. 1(b) shows its demodulated wrapped phase. Figures 1(c) and 1(d) show the two centered first-order differences $I_{x}$ and $I_{y}$ along the $x$ and $y$ coordinates, respectively. The fringe orientation angle modulo $\pi$ is shown in Fig. 1(e); the darker gray level corresponds to $0 \mathrm{rad}$, and the lighter

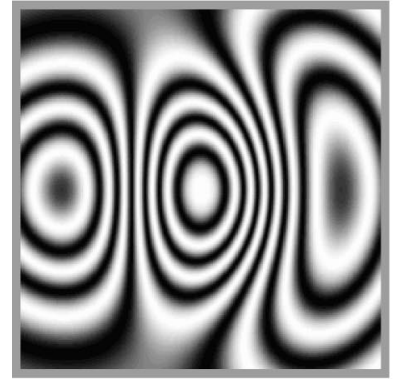

(a)

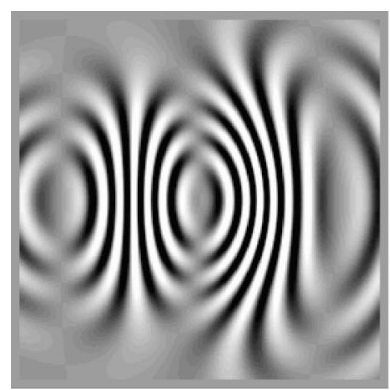

(c)

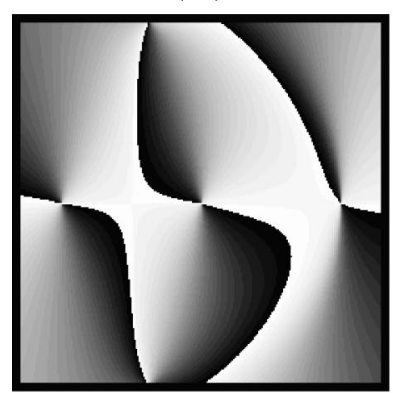

(e)

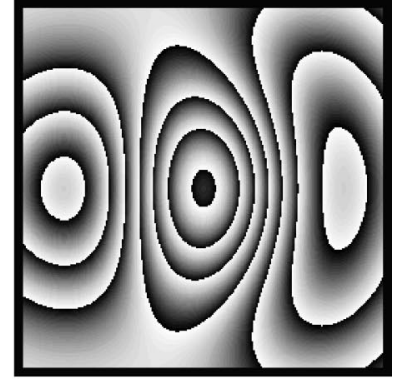

(b)

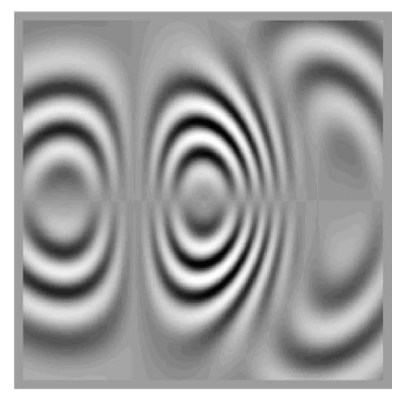

(d)

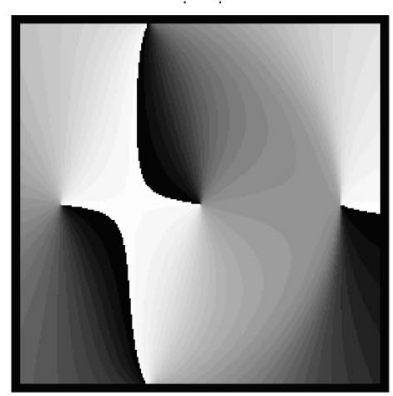

(f)
Fig. 1. Demodulation steps that use the algorithm proposed in Subsection 4.A applied to a noiseless computer-generated interferogram. (a) Fringe pattern, (b) recovered phase, (c) and (d) the two components of $\nabla I$, (e) fringe orientation angle modulo $\pi$, (f) fringe orientation angle modulo $2 \pi$.

gray level corresponds to $\pi$ rad. This is the orientation angle that is directly observable from the interferogram $I(x, y)$. This orientation angle is wrapped modulo $\pi$, which means that the fringe's orientation angle will have the same value at two points situated near and symmetrically away from the center of a given set of closed fringes. Its unwrapped fringe orientation angle (modulo $2 \pi$ ) is shown in Fig. 1(f), obtained with the method proposed by Quiroga et al. ${ }^{10}$ As can be seen from Fig. 1(e), this fringe orientation angle cannot be unwrapped by use of pathindependent unwrapping techniques because the orientation modulo $\pi$ has three essential phase discontinuities. ${ }^{13}$ These discontinuities must be preserved, and that is why an unwrapping strategy that follows the fringes is the best way to unwrap this signal.

Our next example is the demodulation of an experimentally obtained fringe pattern that is shown in Fig. 2(a). These fringes were obtained from a shearing interferometer by use of electronic speckle-pattern interferometry (ESPI). Figure 2(b) shows the fringe orientation modulo $2 \pi$ that was obtained by unwrapping the orientation modulo $\pi$ estimated from the ESPI image. The angle's 
orientation unwrapping was achieved by use of the algorithm by Quiroga et al. ${ }^{10}$; that is, the fringe orientation was unwrapped following the fringes of the interferogram. Figures 2(c) and 2(d) show the signals obtained by use of first-order centered differences to approximate the gradient $\nabla I(x, y)$. Figure 2(e) shows the recovered $\mathbf{n}_{\phi}$

$\nabla I$ signal. Although this signal is orthogonal to the fringe pattern in Fig. 2(a), it is not everywhere in quadrature with it. As consequence, the recovered phase from Figs. 2(a) and 2(e) will have some small distortion that can be removed with the Halley algorithm discussed in Subsection 4.A to finally obtain the phase shown in Fig. 2(f).

Finally, we have calculated the quadrature signal of the noiseless computer-generated fringe pattern $\sin \left(x^{2}+y^{2}\right)$ [i.e., $\cos \left(x^{2}+y^{2}\right)$ ], using the method of Subsection 4.A to compare it with the vortex operator proposed by Larkin et $a l .{ }^{5}$ and the quadrature operator using the Schlieren filter [Eq. (46)]. In Fig. 3(a) we show the ideal quadrature signal. Figure 3(b) shows the quadrature signal ob-

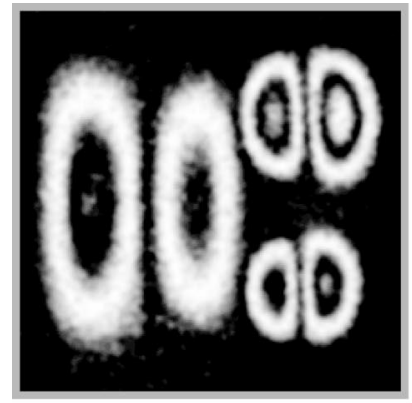

( a )

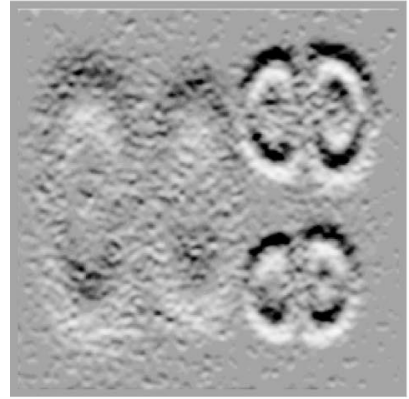

(c)

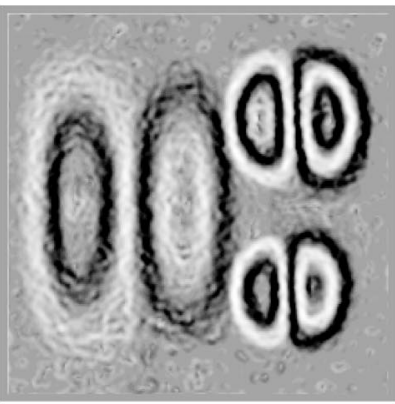

(e)

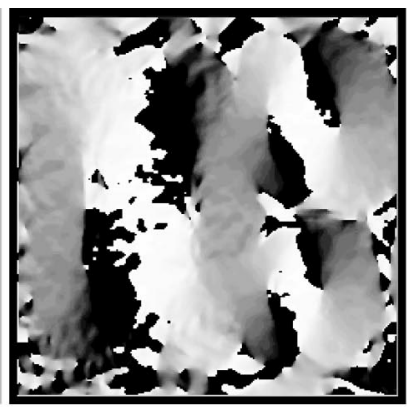

(b)

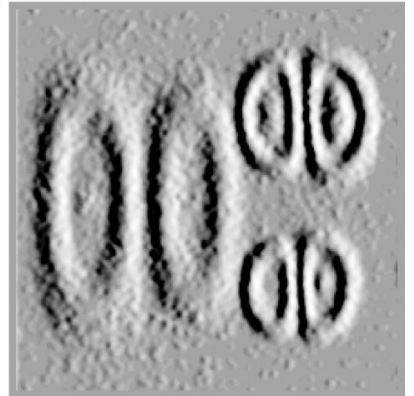

(d)

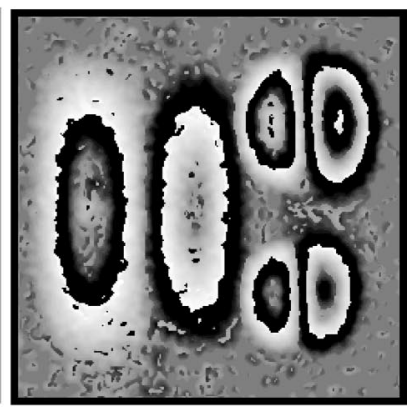

(f)
Fig. 2. Demodulation of an experimentally obtained specklegram. (a) The fringe pattern; (b) the estimated fringe orientation angle modulo $2 \pi$; (c) and (d) the two components of $\nabla I$; (e) the recovered signal $\mathbf{n}_{\phi} \cdot \nabla I$; (f) the recovered phase after the pixelwise adjustment by use of the Halley method.

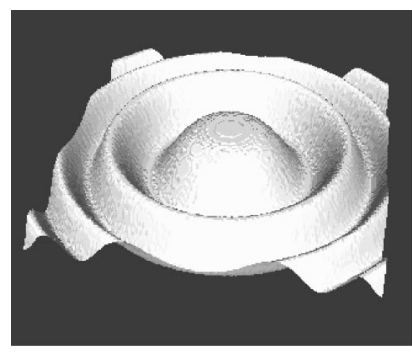

(a)

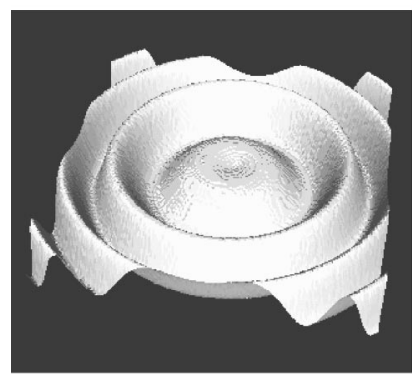

(c)

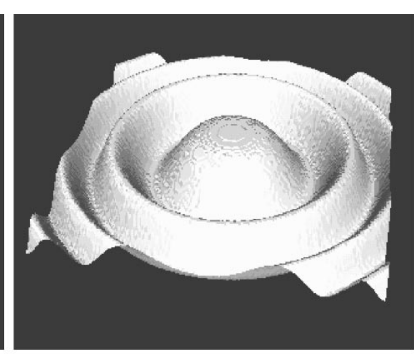

(b)

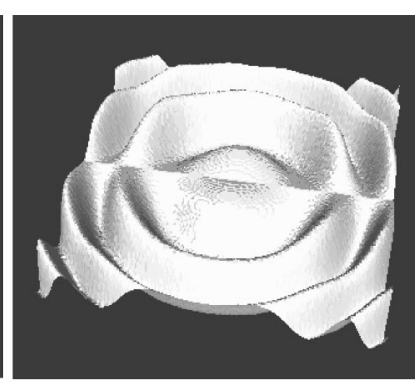

(d)
Fig. 3. Errors obtained with the vortex operator against the demodulation algorithm proposed by use of a noiseless computergenerated interferogram. (a) Desired quadrature signal, (b) quadrature signal obtained with the numerical algorithm proposed in Subsection 4.A, (c) quadrature signal obtained with the vortex operator, (d) quadrature signal obtained by use of the operator of Eq. (46).

tained with the numerical algorithm outlined in Subsection 4.A; one can see that this result is identical to the expected quadrature signal because of the pointwise adjustment by use of the Halley recursion formula. As one can see, the vortex operator distorts the recovered phase [Fig. 3(c)] on regions of very low spatial frequency. Finally, Fig. 3(d) shows the quadrature operator that uses the Schlieren filter; this quadrature formula renders the lowest quality result for the reasons explained above. Note that it may also be possible to construct a hybrid algorithm that uses the vortex operator in a first step, followed by the Halley pointwise adjustment. This algorithm should also give a distortion-free reconstruction.

\section{CONCLUSIONS}

We have presented a quadrature operator that, when applied to a single interferogram, gives its quadrature image $Q\{b \cos (\phi)\}=-b \sin (\phi)$. This result holds true even when the spectrum of the fringes is not well separated, i.e., for single closed-fringe interferograms. We have also shown that this quadrature operator $Q\{I\}$ can be expressed as the vectorial inner product of two vector fields. One of them, i.e., $\nabla I /|\nabla \phi|$, may be regarded as an $n$-dimensional generalization of the Hilbert transform $\mathbf{H}_{n}\{\cdot\}$ of the interferogram, and the other one is the orientation of the fringes $\mathbf{n}_{\phi}=\nabla \phi /|\nabla \phi|$. In this way, our quadrature operator may be expressed as $Q\{I\}$ $=\mathbf{n}_{\phi} \cdot \mathbf{H}_{n}\{I\}$ in any dimension.

We have also pointed out that the critical step in obtaining the quadrature of the interferogram $Q\{I\}$ is the estimation of the fringe orientation $\mathbf{n}_{\phi}=\nabla \phi /|\nabla \phi|$. This 
operator can only be obtained modulo $\pi$ directly from the interferogram irradiance because the cosine function wraps the required signal $\phi$. Given that the wrapped orientation modulo $\pi$ has essential discontinuities that must be preserved, path-dependent unwrapping processes are necessary to obtain the fringe's orientation modulo $2 \pi$.

Regarding $\mathbf{H}_{n}\{\cdot\}$, we have presented two practical algorithms for its estimation: One is a frequency-domain method, which is closely related to the spiral operator presented in Ref. 5, and the other is a space-domain method based on a pixelwise correction of a phase estimate that is directly obtained from the image gradient. We have shown experimentally that this second method works quite well, even in the presence of moderately high measurement noise (e.g., the ESPI image of Fig. 2), and has the additional advantage of avoiding the phase distortions that frequency-domain methods introduce in places where low frequency dominates.

\section{APPENDIX A}

In this appendix we prove in an intuitive way the following approximation:

$$
H\left[\omega_{x}(x, y), \omega_{y}(x, y)\right] I(x, y) \approx \mathcal{F}^{-1}\{H(u, v) \hat{I}(u, v)\},
$$

where $\hat{I}(u, v)=\mathcal{F}\{I(x, y)\}$ is the 2D Fourier transform of $I(x, y)$ of the fringe pattern given by

$$
I(x, y)=b(x, y) \cos [\phi(x, y)]
$$

and the signals $\omega_{\mathrm{x}}(x, y)$ and $\omega_{y}(x, y)$ are the local spatial frequencies of the fringe pattern given by

$$
\omega_{x}(x, y)=\frac{\partial \phi(x, y)}{\partial x}, \quad \omega_{y}(x, y)=\frac{\partial \phi(x, y)}{\partial y}
$$

Let us start by considering the following fringe-signal decomposition as a sum of mutually exclusive squared regions and the local phase $\phi(x, y)$ expanded as a linear phase by use of Taylor-series expansion. This gives

$b(x, y) \cos [\phi(x, y)]$

$$
\begin{aligned}
& \approx \sum_{n, m} \sqcap(x-n, y-m) b(n, m) \cos [\phi(n, m) \\
& \left.\quad+\omega_{x}(n, m)(x-n)+\omega_{y}(n, m)(y-m)\right],
\end{aligned}
$$

where the symbol $\sqcap(x-n, y-m)$ is a squared window, centered at coordinates $(n, m)$, that equals 1 inside the window and zero otherwise. The value $b(n, m)$ is the fringe contrast evaluated at $(n, m)$; the argument of the cosine function is a phase plane $p(x, y, n, m)$ oriented according to $\left[\omega_{x}(n, m), \omega_{y}(n, m)\right]$ within the spatial region around $(n, m)$ and limited by $\sqcap(x-n, y-m)$. If we pass this signal through a linear spatial filter having a frequency response $H(u, v)$, we may write its response approximately as

$$
\begin{aligned}
\mathcal{F}^{-1} & {[H(u, v) I(u, v)] } \\
\approx & \sum_{n, m} \sqcap(x-n, y-m) b(n, m) H\left[\omega_{x}(n, m), \omega_{y}(n, m)\right] \\
& \quad \times \cos [p(x, y, n, m)],
\end{aligned}
$$

where $p(x, y, n, m)$ equals the linear Taylor expansion of $\phi(x, y)$ around the point $(n, m)$ :

$$
\begin{aligned}
p(x, y, n, m)= & \phi(n, m)+\omega_{x}(n, m)(x-n) \\
& +\omega_{y}(n, m)(y-m) .
\end{aligned}
$$

An intuitive explanation of this is that inside of a given squared window $\sqcap(x-n, y-m)$ one has a spatially monochromatic fringe pattern, so its filtered amplitude depends only on its spatial-frequency content along the $x$ and $y$ directions $\left(\omega_{x}, \omega_{y}\right)$. Therefore

$$
\mathcal{F}^{-1}\{H(u, v) \mathcal{F}[I(x, y)]\} \approx H\left[\omega_{x}(x, y), \omega_{y}(x, y)\right] I(x, y) .
$$

This result is exact only if the fringe pattern is just a modulated phase plane. Conversely, this result is less accurate when the modulated phase $\phi(x, y)$ changes abruptly within the domain of interest. This result may not surprise us given that this is the principle behind analog electronic spectrum analyzers and old broadcast FM demodulators that used passive RLC electrical networks such as the balanced slope FM detector. ${ }^{15}$

\section{ACKNOWLEDGMENTS}

J. L. Marroquin and M. Servin were partially supported by grants 34575A and 33429-E from Consejo Nacional de Ciencia y Tecnología, Mexico.

M. Servin can be reached by e-mail at mservin@cio.mx.

*On sabbatical leave at the Centro de Investigaciones en Matematicas, Mexico.

\section{REFERENCES}

1. T. Kreis, "Digital holgraphic interference-phase measurement using the Fourier-transform method,"J. Opt. Soc. Am. A 3, 847-855 (1986).

2. M. Servin, J. L. Marroquin, and F. J. Cuevas, "Fringefollowing regularized phase tracker for demodulation of closed-fringe interferogram," J. Opt. Soc. Am. A 18, 689-695 (2001).

3. J. L. Marroquin, M. Servin, and R. Rodriguez-Vera, “Adaptive quadrature filters and the recovery of phase from fringe pattern images," J. Opt. Soc. Am. A 14, 1742-1753 (1997).

4. J. L. Marroquin, R. Rodriguez-Vera, and M. Servin, "Local phase from local orientation by solution of a sequence of linear systems," J. Opt. Soc. Am. A 15, 1536-1543 (1998).

5. K. G. Larkin, D. J. Bone, and M. A. Oldfield, "Natural demodulation of two-dimensional fringe patterns. I. General background of the spiral phase quadrature transform," J. Opt. Soc. Am. A 18, 1862-1870 (2001).

6. M. Takeda, H. Ina, and S. Kobayashi, "Fourier-transform method of fringe-pattern analysis for computer-based topography and interferometry," J. Opt. Soc. Am. 72, 156-160 (1982).

7. R. N. Bracewell, The Fourier Transform and Its Applications (McGraw-Hill, New York, 2000). 
8. M. Born and E. Wolf, Principles of Optics, 6th ed. (Pergamon, New York, 1980).

9. E. Stein and G. Weiss, Introduction to Fourier Analysis on Euclidian Spaces (Princeton U. Press, Princeton, N.J., 1971).

10. J. A. Quiroga, M. Servin, and F. J. Cuevas, "Modulo $2 \pi$ fringe orientation angle estimation by phase unwrapping with a regularized phase-tracking algorithm," J. Opt. Soc. Am. A 19, 1524-1531 (2002).

11. D. Richards, Advanced Mathematical Methods with Maple (Cambridge U. Press, Cambridge, UK., 2002).

12. D. C. Ghiglia and L. A. Romero, "Robust two-dimensional weighted and unweighted phase unwrapping that uses fast transforms and iterative methods," J. Opt. Soc. Am. A 11, 107-117 (1994).

13. D. C. Ghiglia and M. D. Pritt, Two-Dimensional Phase Unwrapping, Theory Algorithms, and Software (Wiley, New York, 1998)

14. M. Servin, F. J. Cuevas, D. Malacara, and J. L. Marroquin, "Phase unwrapping through demodulation using the regularized phase-tracking technique," Appl. Opt. 38, 19341940 (1999).

15. M. Schwartz, Information Transmission Modulation and Noise (McGraw Hill, New York, 1980). 